\title{
Distribution de cash-flows aux actionnaires : moyens, déterminants, effets et modèles
}

\section{ESJ Social Sciences}

\section{Nabaoui Ahmed}

Chercheur à l'université Hassan II, laboratoire de performance économique et logistique (LPEL), Mohammedia Maroc

\section{Benhrimida Mohamed}

Professeur d'enseignement supérieur Université Hassan II, Laboratoire de Recherche en performance économique et logistique (LPEL), Mohammedia Maroc

Submitted: 18 September 2020

Accepted: 17 November 2020

Published: 31 December 2020

Corresponding author:

Nabaoui Ahmed

DOI: 10.19044/esj.2020.v16n34p230

(c)

Copyright 2020 Nabaoui A. \& Benhrimida M Distributed under Creative Commons BY-NC-ND 4.0 OPEN ACCESS

\section{Resume}

La problématique de la politique de distribution des cash-flows a suscité l'intérêt de plusieurs économistes au fil du temps. Dans les dernières décennies, plusieurs études théoriques mais aussi empiriques, se sont concentrées sur l'objectif d'augurer le mystère de ce " puzzle » tel que le qualifie Black en 1976. Le développement de la finance d'entreprise et de marché a enrichi le terrain théorique et empirique en la matière. Cet article a pour objectif d'examiner les effets de la structure de propriété du capital et la nature d'actionnariat sur le dividende au travers la mise en exergue des différents conflits qui peuvent surgir entre les différentes parties prenantes de la firme, tout en faisant appel à la théorie d'agence, d'asymétrie d'information ou des signaux, de clientèle fiscale, de oiseau en main, d'enracinement, et ce après mise en évidence, des différents moyens de distribution, et de modèles retraçant les effets du montant du dividende sur la valeur de la firme.Ce travail révèle qu'un consensus sur la problématique du dividende est encore loin d'avoir le jour et les chercheurs sont toujours sur la locomotive d'expliquer le dividende chacun au moins à partir de ses constats contextuels.

Mots-clés : dividende, rachat d'action, structure d'actionnariat, théorie d'agence, théorie des signaux, conflit d'intérêt 


\title{
Distribution of Cash Flows to Shareholders: Means, Determinants, Effects and Models
}

\begin{abstract}
Nabaoui Ahmed,
Chercheur à l'université Hassan II, laboratoire de performance économique et logistique (LPEL), Mohammedia Maroc

Benhrimida Mohamed,

Professeur d'enseignement supérieur Université Hassan II, Laboratoire de Recherche en performance économique et logistique (LPEL), Mohammedia Maroc
\end{abstract}

\begin{abstract}
The issue of the cash flows distribution policy has aroused the interest of several economists over time. In recent decades, several theoretical but also empirical studies have focused on the objective of predicting the mystery of this "puzzle" as Black describes it in 1976. The development of corporate and market finance has enriched the theoretical and empirical ground in the matter. The objective of this paper is to examine the effects that the structure of the ownership of capital and the nature of shareholding can have on the dividend by highlighting the various conflicts that can arise between the various stakeholders of the firm, while appealing to the theory of agency, asymmetry of information or signals, fiscal clientele, bird in hand, rooting, and this after highlighting the different means of distribution, and models tracing the effects of the amount of the dividend on the value of the firm. This work reveals that a consensus on the issue of the dividend is still far from having the day and researchers are still on the locomotive to explain the dividend each at least based on its contextual findings.
\end{abstract}

Keywords: Dividend, share buyback, shareholding structure, agency theory, signal theory, conflict of interest

\section{Introduction}

La thématique du dividende n'a pas encore fait l'objet d'un consensus dans la finance d'entreprise, bien qu'elle ait fait l'objet de plusieurs recherches, menées par des chercheurs à travers le monde entier, surtout à partir des travaux de Lintner (1956) etMiller \& Modigliani (1961). Deux décennies après Lintner, Black en (1976), désignait cette problématique de dividende par « dividende puzzle ». Le développement de la finance 
d'entreprise qui s'est encouragé par une richesse théorique (théorie d'agence, théorie du signal, théorie de la clientèle fiscale ...) a permis aux sociétés de recourir à d'autres modes de distribution de cash, tel que le rachat d'actions par la société, qui constitue à son tour un substitut principal du dividende, et ce, suivant des motifs différents, explicites mais parfois implicites. Le choix entre l'un de ces deux moyens de distribution est facteur de plusieurs déterminants, telle que la structure de propriété du capital et la nature d'actionnariat. Étant donné que le dividende est le moyen de distribution des cash le plus fréquent, plusieurs modèles ont été construits visant d'une part, la détermination des principaux facteurs, impactant son niveau de distribution auprès d'une société, ainsi que les effets que peut avoir ce niveau de dividende sur la valeur de l'action en particulier et sur la valeur de la firme de manière générale d'autre part. Cet article vise à :

$\checkmark$ Mettre en évidence les principaux moyens de distribution de liquidité offerts aux sociétés

$\checkmark$ Clarifier les interactions qui peuvent s'établir entre le dividende et la structure de la propriété du capital ainsi que la nature d'actionnariat

$\checkmark$ Déterminer les principaux modèles des effets que peut avoir le dividende sur la valeur de la firme

Le reste de cet article consiste à analyser le rôle du montant du dividende, dans les conflits d'intérêts qui peuvent avoir lieu entre les différentes parties prenantes de la firme (actionnaires, dirigeants et créanciers), en se référant en grande partie à la théorie d'agence et la théorie du signal, avec en fin une conclusion.

\section{Les modes de distribution de cash-flows aux actionnaires}

Lorsqu'une entreprise dispose de flux de trésorerie élevés, elle doit décider de les affecter. Deux possibilités lui sont offertes : si l'entreprise dispose d'opportunités d'investissement rentables, elle peut réinvestir ces liquidités dans des projets générateurs de croissance, c'est généralement la solution la plus retenue par les entreprises jeunes en forte croissance, qui pratique une forte rétention du bénéfice. Cependant en l'absence d'opportunités à financer, ou en présence de flux de trésorerie excédentaires vis-à-vis du besoin d'investissement, tel est le cas de la plupart des entreprises matures, ces flux peuvent être conservés en trésorerie ou distribuées aux actionnaires. En réalité la décision de retenir ou distribuer les cash-flows se trouve fortement influencée par les imperfections du marché : coût de transaction et asymétrie d'information, conflit d'agence, imposition du dividende périodique et du dividende synthétique (plus-value en capital), enracinement des dirigeants, etc. 
En 1976, quand il parlait de la complexité de comprendre le dividende, Black, écrit «the harder we look at the dividend picture, the more it seems like a puzzle, with pieces that just don't fit together », ce qui peut se traduire par: «Plus nous examinons l'image du dividende, plus cela ressemble à un casse-tête, avec des pièces qui ne correspondent tout simplement pas ». Black montrait que le niveau de distribution n'est pas une chose évidente, comme il l'affirmait c'est un puzzle difficile à maitriser, d'où l'importance des études qui portaient sur ce sujet et qui ne cessent à nos jours d'essayer d'appréhender les motifs de la préférence pour la distribution ou pour la rétention. Connue souvent en finance sous l'appellation politique de dividende, qui peut être définie comme étant l'arbitrage entre la rétention de bénéfices d'une part, et le versement de liquidités aux actionnaires accompagné de l'émission de nouvelles actions d'autre part, en fait, cet arbitrage ne doit pas être observé sur une courte période, car les entreprises qui versent des dividendes ne font pas des augmentations de capital tous les ans, mais plutôt dans une perspective à moyen ou long terme (Albouy, M. 1990).

\section{Le dividende}

Les dividendes constituent une affectation possible des flux de trésorerie disponibles dans l'entreprise et qui proviennent à l'origine d'un résultat bénéficiaire. Ils se traduisent par un transfert de capitaux de l'entreprise vers les actionnaires (Berk\&Demarzo.2011). Le dividende est défini par Frankfurter \& Wood (2003) comme «la distribution de revenus (présents ou passés) en actifs réels aux actionnaires de l'entreprise en proportion de leur participation au capital ». Les dividendes, par référence au code de commerce Marocain, ne peuvent excéder le bénéfice distribuable de l'entreprise tel que prévu par l'article 330 de la Loi $n^{\circ} 17-95$ relative aux sociétés anonymes, qui stipule que le bénéfice distribuable est constitué du bénéfice net de l' exercice, diminué des pertes antérieures ainsi que des sommes à porter en réserve par application de l' article 329 (relative à la réserve légale) et augmenté du report bénéficiaire des exercices précédents. Ce qui permet de dire que le niveau de dividende est en principe déterminé par le bénéfice.

\subsection{Les types de dividendes}

Plusieurs critères peuvent être adoptés pour classer les dividendes, notamment, l'échelle temporelle, le moyen de payement, la source ou l'origine de payement (bénéfice ou liquidation). Ainsi, on peut distinguer trois types, premièrement, Les dividendes en liquide ou en actions supplémentaires : Ce mode augmente le nombre d'actions en circulation et peut contribuer à la diminution du cours des actions, la distribution de cash-flows aux actionnaires 
ne peut se faire que par l'un des deux moyens : le dividende classique et le rachat d'actions. Deuxièmement, Les dividendes réguliers et les dividendes exceptionnels : Les dividendes réguliers sont payés à intervalle temporel régulier, la plupart des firmes américaines versent des dividendes chaque trimestre, Cependant, dans d'autres pays du monde le versement se fait de manière semi annuelle ou annuelle (Damodaran, 2010). Ainsi au Maroc le versement est annuel. Les dividendes irréguliers sont versés à intervalle irrégulier, certaines entreprises, peuvent distribuer des liquidités qui excèdent leur bénéfice non distribué, ce type de dividende est appelé dividende de liquidation, considéré par le fisc américain comme plus-value en capital et non comme un revenu ordinaire, en France est assimilé fiscalement à un dividende et au Maroc selon Abou El jaouad. (2010) : « sont considérés comme des produits financiers les sommes prélevées sur les bénéfices ou les réserves pour l'amortissement du capital ou le rachat d'actions et les bonis de liquidation augmentés des réserves distribuées ainsi que les tantièmes ordinaires perçus de l'affectation du résultat, tous ces produits, de ce fait, ils sont totalement exonérés par similitude aux dividendes ». Troisièmement, Les « vrai-faux dividendes » : Ce sont des rémunérations (salaires plus élevés, avantages en nature ...) en faveur de certains actionnaires majoritaires, généralement des dirigeants, ce type de rémunérations trouve sa justification dans les conflits d'agence qui seront traités dans les parties à venir (Damodaran, A, 2010).

\subsection{Les mesures de la politique de dividende}

Parmi les plus importantes mesures de la politique de dividendes, on peut calculer : le rendement de l'action et le ratio de distribution de dividendes (Damodaran, A, 2010).

$$
\text { Le rendement de l'action }=\frac{\text { montant annuel de dividendes versés par action }}{\text { cours de l'action }}
$$

Il s'agit de l'évaluation de la rentabilité du dividende dans la rentabilité globale d'une action, cette mesure est significative dans la mesure où elle contribue à la détermination du rendement global de l'action, qui s'ajuste par l'addition de la plus-value à cette mesure. La seconde mesure largement utilisée, dans le cadre d'analyse des politiques de dividendes est le ratio de distribution de dividendes (Turki. 2013), elle se calcule ainsi :

$$
\text { Le ratio de distribution de dividendes }=\begin{gathered}
\text { dividendes } \\
\text { bénéfices }
\end{gathered}
$$

Vernimmen. (2014), renonce au premier taux (le rendement de l'action) et le remplace - tout en conservant la seconde mesure à savoir le taux de distribution- par le taux de croissance du dividende par action. En se limitant uniquement à ces deux critères pour analyser la politique de dividendes, il confirme : « tout autre critère est non pertinent, souvent inexact, voire aberrant. Ainsi, il est absurde de ramener le dividende au nominal d'une 
action, puisque ce dernier est très souvent déconnecté de la valeur des capitaux propres ». Ainsi, il est difficile pour la firme de fixer un taux de rendement cible pour l'actionnaire, c'est ce dernier qui évalue l'entreprise, donc l'action, et détermine le rendement souhaité et non l'inverse.

\subsection{Les effets de la stabilité et de la variation du dividende}

Plusieurs études affirment l'effet que peut avoir le rythme d'évolution $\mathrm{du}$ dividende, sur le comportement des investisseurs, qui reflète un changement dans la façon dont ils perçoivent la valeur de la firme sujette de ce rythme de distribution, ce paragraphe relève les principales explications fournies par la discipline à l'égard de ce dernier dans deux classes d'analyse, à savoir, la stabilité et la variation du dividende.

\subsubsection{Un dividende stable}

Les firmes ne modifient pas dans une grande mesure le montant des dividendes à distribuer, elles essayent de maintenir un dividende quasi stable, ou d'adopter une augmentation graduelle dans le dividende versé. Cette stagnation s'explique par plusieurs facteurs, notamment, comme premier facteur, la préoccupation des firmes de maintenir leur capacité à pouvoir distribuer des dividendes supérieurs dans les périodes futures, le second facteur provient du fait que les marchés réagissent négativement (baisse du cours de l'action), suite à l'annonce d'une réduction de dividendes (Damodaran. 2010). Il y a aussi le stade de maturité de l'activité de la firme c'est à dire son cycle de vie comme le montre la théorie de cycle de vie des dividendes de Mueller (1972), dont on peut rappeler la portée : les entreprises jeunes à forte croissance réinvestissent en grande partie leurs bénéfices dans des opportunités d'expansion de marché ou des innovations de produits générateurs de rentabilité. A contrario les entreprises au stade de maturité ont épuisé leurs possibilités d'innovation et de croissance, le marché est submergé de concurrents, l'avantage compétitif est érodé. Les couts d'agence nourrissent une forte exigence d'un tel taux de rendement, ce qui conduit les entreprises matures à distribuer le cash excédentaire, ensuite, le contexte économique $^{1}$, fiscal ${ }^{2}$ de chaque pays, ainsi en Tunisie selon Taleb (2015),

${ }^{1}$ La stabilité des dividendes, ou le cas échéant l'augmentation graduelle dans les dividendes versés, peut constituer un argument important pour une plus grande mobilisation de l'épargne vers le renforcement des capitaux propres. Cette stabilisation semble être une contrainte pour les entreprises tunisiennes cotées qui cherchent dans un contexte national à inciter l'épargnant de mobiliser ses fonds vers les marchés financiers à travers des placements à revenu raisonnable et immédiat. Taleb, L. (2015).

${ }^{2}$ Les deux auteurs (Adjaoud, \& Zéghal 1993) Ont vérifié si l'exonération fiscale adoptée par le gouvernement canadien en 1985 en faveur de ses investisseurs( jusqu'à hauteur cumulative de 500.000\$ de gain en capital ), ils ont obtenu de résultats significatifs compatibles avec l'hypothèse que les entreprises avaient abaissé leur taux cible de distribution en dividendes. 
L'évolution du dividende par action (DPA) par rapport au bénéfice par action (BPA) montre que $75 \%$ des entreprises Tunisiennes essayent d'adopter une politique de dividende stable ou le cas échéant une augmentation graduelle dans le temps.

Lintner (1956) dans son modèle d'ajustement partiel de la politique de dividende, montre que les dirigeants associent le changement de la politique de dividende au ratio de distribution antérieur, ainsi qu'au bénéfice actuel tout en préservant leur réticence à la baisse du dividende et en considérant leur taux de distribution comme cible à long terme. Il suggère la relation suivante pour la description de l'évolution du dividende d'une firme $\mathrm{i}$ :

$\Delta \mathrm{Di}, \mathrm{t}=\mathrm{ai}+$ bi ri EPSi, t - bi Di,t-1.

$\Delta$ Di,t est la variation du dividende entre $\mathrm{t}-1$ et $\mathrm{t}$.

Di,t-1 est le dividende versé à $\mathrm{t}-1$.

EPSi,t est le bénéfice courant.

ai est la constante représentative de la résistance à la réduction du dividende,

ri est le taux de distribution cible de la firme,

bi est la vitesse d'ajustement du dividende.

Ce modèle révèle la réactivité de la firme à toute circonstance qui pourrait affecter sa politique du dividende dans l'objectif de préserver sa stabilité de distribution. (Turki. 2013).

\subsubsection{Un dividende variable}

En finance moderne (finance d'entreprise ou de marché), le risque est assimilé à l'incertitude ou à la probabilité de percevoir un revenu négatif (une perte), positif (gain) ou nul différent de celui espéré (Damodaran, 2007). Le risque est alors attaché à l'impossibilité de prévoir les réalisations éventuelles de sortie d'une décision. Autrement dit, la prise de risque se réfère à des actions, à conséquences incertaines entreprises par un décideur (dirigeant d'entreprise ou investisseur), (Yessoufou. 2018). Toute variation du dividende est perçue par le marché comme une information sur la façon dont les managers évaluent leurs conceptions quant à l'avenir de la firme, notamment le niveau futur de ses flux monétaires et leur variabilité. Une diminution du niveau ou une variabilité accrue des flux attendus, peut motiver les managers à réduire ou à supprimer le dividende, tandis qu'une augmentation de niveau futur de flux monétaires ou une variabilité amoindrie justifierait sa hausse. De ce qui précède, la variation du dividende peut renseigner aussi bien une information sur la variation du niveau des flux futurs, ainsi que leur variabilité et par conséquent une information sur le risque de la firme. Les écrits 
pertinents de signalisation estiment que le niveau optimal du dividende soit négativement relié à la volatilité du rendement, donc au risque total de la firme (Bar -Yousef et Huffman 1986, Lapointe, 1995) ou à son risque Béta (Kale et Noe 1990), cité dans Atindéhou (1996). A toute variation inattendue du dividende correspond une variation du risque dans le sens contraire, ainsi pour une volatilité accrue des flux, le rendement de la firme devient plus incertain ainsi que son risque, et pour ne pas supporter des charges financières suite à un endettement externe, l'entreprise, doit réduire la distribution de dividende afin de garder un niveau de ses financements propres, par conséquent, la baisse inattendue du dividende peut signaler une augmentation du risque total de la firme et vice-versa pour une hausse inattendue du dividende.

Selon (Fodil et al, 2005), la firme bien établie ne révise son dividende qu'avec prudence, tant aux USA (Lintner, 1956 ; Baker et al., 1985) qu'au Canada (Adjaoud, 1986). Une révision inattendue, donc surprenante, peut alors signaler que la firme entrevoit un net changement dans la fraction dite permanente de ses rentrées, soit ce coussin sur lequel elle compte pour financer des projets et verser des dividendes réguliers. L'on sait toutefois que la correction boursière de même sens constitue une condition première mais insuffisante pour soutenir l'hypothèse d'information.

Le rythme de distribution des dividendes varie d'un pays à l'autre et reste inhérent aux spécificités fiscales, aux niveaux de croissance des firmes et aux degrés de mise en place des principes de la bonne gouvernance.

Dans son étude empirique menée sur les entreprises américaines d'un échantillon de 738 hausses contre 651 baisses de dividende, (Atindéhou 1996) a conclu ce qui suit :

$>$ Toute variation positive /négative du dividende, s'accompagne en moyenne d'un changement négatif/positif du risque béta.

$>$ La hausse du béta après une baisse du dividende régulier demeure significative, ce qui n'est pas le cas pour un échantillon neutre. Il semble donc que la baisse du dividende contrairement à la hausse, véhicule de l'information sur le risque.

\section{Le rachat d'actions}

Le rachat d'actions, est une opération par laquelle une entreprise, procède à l'achat de ses propres actions. Plusieurs études théoriques (l'effet de la clientèle fiscale, la régulation de la sous-évaluation du cours, la recherche d'une structure financière optimale), et empiriques se sont efforcées pour expliquer les motivations qui poussent les entreprises à adopter cette pratique financière, qui a connu son existence dés le début des années 1980 dans les pays Anglo-saxon, notamment les Etats-Unis et le Canada. Ainsi, entre 1985 et 1996, les montants des rachats d'actions aux États-Unis ont augmenté de $650 \%$ comparativement à $50 \%$ pour les dividendes (Albouy \& Morris 2006). 
En termes monétaires, les rachats d'actions représentent l'équivalent du versement de dividendes (Jiraporn, 2006) ${ }^{3}$. La législation Marocaine n'a reconnu légale, cette pratique financière de manière symétrique aux autres législations qu'à partir de 2003 et de façon plus pratique en 2008, et ce à travers l'article 281 de la loi 17-95 relative à la société anonyme en modifiant et en complétant l'article 280 de cette même loi, ainsi l'article 281 stipule : «Par dérogation aux dispositions du paragraphe 1 de l'article 280, les sociétés dont les titres sont inscrits à la cote de la bourse des valeurs peuvent acheter en bourse leurs propres actions, en vue d'assurer l'animation du marché des dites actions, telle que requise par les dispositions de l'article 14 du dahir portant loi n ${ }^{\circ} 1-93-211$ relatif à la bourse des valeurs, ou pour tous autres motifs fixés par le conseil déontologique des valeurs mobilières, dans le respect des exigences de transparence et de bon fonctionnement du marché $\gg$.

Il existe deux grandes catégories de rachat, la première concerne le rachat à un prix supérieur au prix du marché, tandis que la seconde renvoie au rachat à un prix égal au prix du marché, suivant différents motifs.

\subsection{Les motifs du rachat d'actions}

\subsubsection{Les motifs d'ordre économique et social}

Plusieurs études (Dann 1981, Jensen 1986, Weisbenner 2000, Kahle 2002, Bens et al 2003) ont cherché à expliquer le recours des firmes à la politique de rachat d'actions, dans différents pays du monde. Le rachat d'actions peut être considéré comme un élément régulateur d'un processus d'allocation optimale des ressources sur le marché tout en permettant aux actionnaires, d'investir leurs fonds en surplus dans des activités ayant de forts besoins aux fonds propres (Akrawati 2001). Dans un sens large, le rachat d'actions poursuit deux objectifs majeurs, il s'agit du maintien et de l'amélioration du couple personnes / finance, au travers la recherche de la cohésion sociale et la gestion du capital. S'agissant des motifs de cohésion sociale, c'est l'achat en cas de refus d'agrément, ainsi dans les sociétés familiales ou semi-politiques (par exemple celles qui ont pour objet la publication des revues et journaux), les statuts prévoient des clauses d'agrément de cession accompagnées des clauses de préemption afin d'éviter que l'actionnaire devienne prisonnier de ses actions. D'autre part, le rachat peut être effectué par les sociétés en vue de l'attribution à leurs salariés, afin de les aligner sur les objectifs au même titre que les actionnaires. En ce qui concerne Les motifs d'ordre économique, C'est la réduction du capital non motivé par les pertes, cette technique suppose l'existence des bénéfices ou des

${ }^{3}$ Albouy, M., \& Morris, T. (2006). Les rachats d'actions au Canada : motivations et impact de l'activité économique. Finance Contrôle Stratégie, 9(4), 5-32. 
réserves suffisants afin que cela ne se fasse au détriment des créanciers de l'entreprise, «Elles consistent, pour l'essentiel, à éliminer des charges inutiles » (Akrawati 2001), c'est un amortissement du capital. Ainsi les sociétés peuvent gérer leurs capitaux en procédant, dans des situations de crises ou en cas de manœuvres hostiles d'offre publique d'achat (OPA), menées par des actionnaires de bloc, en intervenant sur le marché par des annonces dans la volonté de racheter leurs propres actions afin d'éviter des chutes de cours.

\subsubsection{Les autres motifs de rachat d'actions}

a) La régulation du cours

La société (les insiders) est mieux informée sur son activité que toute autre partie prenante, notamment ses actionnaires, lorsqu'elle constate que ses titres sont moins évalués, elle propose un cours d'achat supérieur au cours du marché au travers une OPA. Elle cherche donc une réaction du cours à la hausse, c'est une autre forme des signaux financiers à côté de celle de l'augmentation du dividende que peut pratiquer la société. Il faut mentionner que le recours à la signalisation par le rachat est de plus en plus pratiqué dernièrement que la signalisation par l'augmentation du dividende. Dans sens, selon une étude empirique, réalisée par Grullon et Michaely (2002) sur le marché Américain ${ }^{4}$; de 1974 à 1998 le ratio de distribution moyen des dividendes passe de $22,3 \%$ à $13,8 \%$, alors que celui des rachats passe de $3,7 \%$ à $13,6 \%$ sur la même période. Cependant selon une étude menée par (El Houcine \& Boubaker 2015) sur les entreprises suisses, le rachat d'actions ne conduit pas toujours à une hausse du cours de l'action, il s'agit selon ces auteurs " d'un jugement hâtif et myope, car mal documenté ».

\section{b) L'effet fiscal}

Le rachat d'actions par l'entreprise de ses propres actions peut être, entre autres, motivé par sa volonté de prendre en considération le niveau de l'impôt que vont supporter ses actionnaires à l'encaissement des dividendes ou à la vente de leurs actions, de telle sorte qu'elle opte toujours pour la solution la plus avantageuse à ses propriétaires, d'une part. D'autre part, pour les firmes soumises à l'impôt sur les bénéfices, le recours à l'endettement, génère une économie d'impôt suite à la déductibilité des charges financières, dans ce sens la société doit chercher un gain fiscal en renforçant les dettes au détriment des capitaux propres en procédant au rachat d'actions. Toutefois cette solution ne va pas sans effets, car elle peut conduire comme le prévoient Modigliani et Miller à une hausse du ratio du levier, ce qui pourra engendrer une baisse du cours de l'action.

\footnotetext{
${ }^{4}$ Cité dans Lamyaa, R. I. A. D., \& TOUILI, K. (2017). Pourquoi choisir le rachat d'actions?
} Revue du contrôle, de la comptabilité et de l'audit, 1(3). 


\section{c) Des free cash-flow (flux monétaires excédentaires)}

Selon la théorie d'agence et du portefeuille les arguments qui peuvent pousser l'entreprise à procéder au rachat d'actions peuvent se résumer ainsi :

$>$ L'entreprise restitue du cash à ses actionnaires tant qu'elle est dans une position dominante sur son marché, et toute tentative d'investissement en achetant des titres d'autres sociétés concurrentes sera refusée par ses actionnaires.

$>$ La société laisse l'initiative de diversification aux actionnaires par le biais du marché boursier.

$>$ En réduisant ses flux de trésorerie, la société prend l'image d'une entité moins riche et moins attrayante pour une éventuelle OPA hostile.

\section{d) Amélioration des ratios financiers}

Suite au rachat d'actions, le nombre d'actions diminue ainsi que les fonds propres, ce qui contribue à la hausse du bénéfice par action et de la rentabilité des fonds propres.

e) Le souci de vouloir échapper à une OPA hostile

Le rachat par l'entreprise de ses propres actions, lui permet d'éviter qu'un acquéreur hostile ne réussisse sa mainmise sur le contrôle de la société.

\section{f) Remédier au problème de dilution lié aux produits dérivés émis} par la société (POA)

Le recours massif au plan d'option sur achat d'action tend à être un élément largement explicatif de la popularité des rachats d'actions que les firmes ne cessent de pratiquer, dans la mesure où les entreprises qui offrent des stock-options pour aligner les intérêts des dirigeants (bénéficiaires) sur ceux des actionnaires, se trouvent dans l'obligation de réguler les problèmes résultants de la dilution du bénéfice par action (BPA) et du droit de vote. Car le nombre d'actions augmente suite à la mise à la disposition des dits bénéficiaires de nouvelles actions, surtout via l'émission de ces dernières après l'exercice des options par leurs bénéficiaires (les salariés), (Yermak, 1995.Palia, 2001) cité dans (Elhoucine, Boubaker), tel qu'il a été affirmé par (Desbrières et al 2000) «On reproche aussi aux POA de nuire aux actionnaires parce qu'ils entraînent une dilution de leurs droits sur la richesse créée et de leurs droits de vote $»$. Donc l'entreprise pour lutter contre cette dilution peut acheter des actions préalablement à l'exercice des options. Ce motif a été confirmé empiriquement, dans le contexte Américain par (Weisbenner 2000, Kahl 2002, Bens et al 2003), et par (Albouy \& Morris 2006) dans le contexte Canadien. Bens et al (2003) mettent un lien positif entre la dilution du BPA suite à l'exercice des stocks options et le rachat d'actions, en confirmant que ce dernier devient de plus en plus important lorsque le niveau du BPA est au dessous du seuil souhaité par les actionnaires. D'autres motifs tels que 
l'enracinement, peuvent être retenus par certains auteurs en tant que principale motivation du recours à l'opération de rachat d'actions.

\subsection{La pratique du rachat d'action par les firmes marocaines}

Le rachat par les entreprises Marocaines de leurs propres actions, est pratiqué ces dernières années par quelques entreprises cotées à la Bourse des Valeurs de Casablanca (BVC), en tant qu'un moyen important dans la régularisation des cours des actions, ainsi qu'un outil moderne dans le cadre de leur mode de gouvernance actionnariale, étant donné son rôle dans la création de la valeur (Benzaouagh \&Tebbaa 2016). La société «Snep » a à titre d'exemple cédé sur le marché de blocs, le 30 janvier 2018, 112.281 actions auto-détenues issues de son ancien programme de rachat, représentant $4,68 \%$ de son capital. Ces actions s'étaient beaucoup dépréciées avant de reprendre au titre de l'exercice 2018. Le recours des entreprises marocaines à la technique de rachat de leurs propres actions, s'est encouragé par la réglementation de 2003, en effet ces firmes actives dans ce sujet cherchent à rendre cette pratique en tant qu'un nouveau mode de gouvernance en favorisant l'actionnariat de leurs salariés, en leur offrant des privilèges (prix avantageux) de participation dans le capital de la société dont ils sont salariés.

\section{Influence de la structure d'actionnariat sur la distribution}

Parler de l'actionnariat revient à désigner la qualité de l'actionnaire, dans la mesure où plusieurs catégories d'actionnaires devront être distinguées. Deux informations permettent de caractériser la structure d'actionnariat, il s'agit de la part détenue par le(s) actionnaire(s) en capital de la société (concentration plus ou moins forte) et de l'identité ou la nature de l'actionnaire. Dans ce paragraphe, à travers la littérature, Les effets de la concentration (1) et de la dispersion du capital (2) sur la distribution seront présentés.

\section{La part du capital détenue par l'actionnaire dans la firme}

Le degré d'implication de l'actionnaire dans le contrôle des dirigeants ne sera pas le même selon sa part détenue dans le capital, ainsi qu'en fonction de ses objectifs et de ses horizons de placement. Un actionnaire détenant une part importante du capital sera de plus en plus incité à s'investir dans la gouvernance de l'entreprise qu'un actionnaire minoritaire. De même la présence d'un actionnaire majoritaire (actionnaire de contrôle) peut agir comme un mécanisme de gouvernance susceptible de discipliner les dirigeants (Dmsetz et Lehn 1985. Shleifer et Vishny 1986) et de renforcer la performance de la firme. Néanmoins, comme le mentionne (Shleifer et Vishny 1997), le pouvoir des actionnaires majoritaires dépend du degré de protection légale de leurs votes (efficaces seulement dans les pays dont le système légal 
est relativement développé). Toutefois l'actionnaire de contrôle peut profiter de sa position et se faire octroyer des rémunérations très avantageuses, et s'attribuer des richesses de la firme au détriment des actionnaires minoritaires et des autres parties prenantes, ce qui peut engendrer une expropriation de l'actionnaire minoritaire suite à un enracinement de l'actionnaire majoritaire, préjudiciable à la performance de l'entreprise à cause de la hausse des coûts d'agence (Shleifer et Vishny 1997 ; Mard, et al. 2014).

\subsection{Le rôle de l'actionnaire principal ou majoritaire dans la distribution}

Le dividende, en tant que partie du bénéfice net exigé et souhaité par l'actionnaire, peut remplir d'autres missions dans le processus de mise en place de certains mécanismes de contrôle des dirigeants. Dans ce sens, Easterbrook (1984) confirme que les grands actionnaires exigent des paiements de dividendes élevés dans le but de réduire le conflit d'agence. Gadhoum (1995) dans une étude sur les entreprises canadiennes démontre que le principal actionnaire exerce une influence prépondérante sur le versement des dividendes réguliers. Shleifer et Vishny (1997) soutiennent que les grands actionnaires préfèrent tirer des avantages privés de l'entreprise et ils ont tendance à préférer une distribution limitée des dividendes, Il s'agit selon ces deux auteurs d'une forme d'expropriation des actionnaires minoritaires.

\subsection{Le rôle des actionnaires minoritaires}

Dans certaines législations, comme celle Suisse, les actionnaires minoritaires détenant au moins $10 \%$ du capital de la société, disposent d'un droit particulier leur protégeant contre les mauvaises pratiques des actionnaires majoritaires, ces règles trouvent leur place généralement dans les lois des sociétés anonymes, ainsi «la loi autorise les sociétés à prévoir des dispositions statutaires particulières afin de protéger certains groupes d'actionnaires » Trigo \& Bahar (2002). Cependant la règle capitaliste impose que la décision revient au pourcentage du capital détenu, d'où la faible influence des minoritaires sur la distribution.

\section{La nature ou l'identité d'actionnaire}

\subsection{L'actionnariat salarial}

C'est un dispositif qui consiste à accorder à un salarié d'une firme la possibilité de devenir un actionnaire, selon différents procédés. C'est un choix stratégique adopté par la gouvernance d'entreprise, qui cherche au travers cette participation du salarié dans le capital de cette dernière, à ce qu'une unification des intérêts de deux parties prenantes déterminantes (apporteurs de capital et apporteurs de travail) dans la vie et dans la croissance de la firme, soit garantie. Et à ce que les conflits entre ces deux parties se trouvent réduits, 
suite à la naissance après cette action, d'une cohésion entre salarié et organisation. Les innovations en management sont toujours associées aux économies libérales, du sens propre du terme, où la principale autorité de l'économie dans des périodes normales est le marché et non pas des formations économiques profitant du népotisme que leur confient les pouvoirs tel est le cas des économies sous-développées. Cela ne doit pas être interprété dans un sens où les économies libérales sont toujours justes et toutes responsables socialement à l'égard de leurs salariés, mais dans le sens où les parties prenantes de ces économies et désignons plus précisément celle salariale, s'affrontent dans un terrain de défense des intérêts de chacune sans interventionnisme apolitisé ou réprimant de cet acteur économique, facteur clés de succès dans toute croissance économique via sa haute productivité, conditionnée par une meilleur perception de milieu ou de l'environnement du travail. Cette nouvelle pratique gouvernementale, à notre stade de connaissance et à notre interprétation s'inscrit dans une perspective de résolution des problèmes, liés aux conflits d'intérêt généralement traités en se référant à la théorie d'agence, surtout la problématique du partage de manière équitable, de la richesse créée entre les acteurs qui en ont contribué. Problématique que les anciennes procédures organisationnelles ou légales tel que le syndicalisme n'ont pas pu résoudre au travers tout le monde. Plutôt ces mécanismes procéduraux-légales n'ont fait qu'encourager les inégalités à creuser davantage entre ces différentes parties prenantes.

La législation marocaine a prévu des dispositions visant à favoriser l'actionnariat salarié avec le Plan d'Epargne Entreprise (PEE), introduit par la Loi de Finances 2013. A titre d'exemple, l'abondement versé par l'entreprise au salarié en vue de l'aider à constituer son épargne ne se substitue pas à un élément de salaire. L'abondement n'est ni considéré comme un complément de salaire, ni imposable sur le revenu sauf lorsqu'il dépasse $10 \%$ du montant annuel du salaire imposable. Aussi, une autre disposition prévue par la loi permet aux salariés d'investir dans le capital de leur entreprise tout en étant exonérés de l'impôt sur les plus-values réalisées.

\subsection{L'actionnariat managérial ; enracinement}

L'analyse de (Fama et Jensen 1983) distingue deux types d'organisations, celles avec séparation de propriété-décision et celles sans séparation propriété-décision, (Lawriwsky. 1984) distingue trois types de firmes : les firmes managériales (dont moins de $10 \%$ du capital sont détenus par les dirigeants), dans ce type de sociétés selon (Charreaux. 1991), la part détenue par les dirigeants n'est pas significative ainsi que la dissociation des organes de propriété et de contrôle est nettement marquée. Dans les sociétés familiales ou à contrôle privé, le capital est détenu par une famille qui désigne un de ses membres en tant que dirigeant, ce qui affaiblira la séparation 
propriété-contrôle et propriété- décision. Dans les sociétés contrôlées (par une autre firme), le capital est concentré, la majeure partie du chiffre d'affaires revient aux représentants de la firme qui contrôlent, et c'est à eux de nommer les dirigeants, ce qui laisse dire formellement qu'il y a une séparation propriété-décision tant que les dirigeants, ne sont pas personnellement propriétaires, d'où une relation d'agence. Cependant en approfondissant l'analyse, il devient possible de comprendre une parfaite dépendance des dirigeants vis-à-vis de leurs actionnaires principaux, d'où la séparation propriétaire-décision est fortement atténuée.

\subsection{L'actionnariat familial}

L'actionnariat familial c'est la forme d'actionnariat la plus répandue au monde (la porta et al 1999) ${ }^{5}$, qu'il s'agisse dans les PME ou dans les grandes entreprises cotées, ainsi aux Etats Unis parmi les 500 grandes sociétés, plus de 35\% sont des sociétés familiales (Shleifer et Vishney 1986; Wang 2006). En France, les deux tiers des sociétés cotées sont des sociétés familiales (Faccio et Lang. 2002). Au Maroc plus de 90\% des entreprises sont familiales, bien que les statistiques relatives à ce sujet soient limitées (Nazih 2014). La théorie d'alignement nous renseigne que l'actionnaire familial privilégiera toute sorte de décisions de nature à créer de la valeur à long terme, cette vision de long terme se concrétise aussi bien au travers une forte rétention motivée par le souci de réinvestir, et donne de la valeur aux liens et relations de cet actionnaire et ses salariés, ainsi qu'aux autres parties prenantes. La bonne réputation est une contrainte qui pèse lourdement sur les comportements de l'actionnaire familial et elle le conduit loin de vouloir s'attribuer des bénéfices privés au détriment des actionnaires minoritaires. Contrairement à la théorie de l'enracinement, l'actionnaire familial peut chercher à conserver le pouvoir afin d'en retirer des bénéfices personnels, le refus de communiquer des informations stratégiques de manière symétrique, ou le refus de mettre en place des structures de gouvernance efficace (séparation du poste de président et du directeur général, hétérogénéité et indépendance des administrateurs ) constitue, à côté d'autres manœuvres, un moyen pour l'actionnaire familial de s'enraciner .

En ce qui concerne le cas marocain, surtout le tissu économique regroupe des entreprises caractérisées par une structure de propriété à majorité familiale, dont les membres sont fortement présents dans la gestion, la concentration de la propriété dans ces entreprises favorise une mainmise de l'actionnaire majoritaire sur le pouvoir. Cette structure explique l'existence d'une corrélation positive entre le dividende et le capital détenu par les administrateurs.

\footnotetext{
${ }^{5}$ Cité dans Mard, Y., Marsat, S., \& Roux, F. (2014).
} 


\subsection{L'actionnariat institutionnel}

Dans un cadre d'actionnariat institutionnel, le contrôle de la société se trouve réduit à la variation du prix de l'action, en raison du faible gain que peut attendre cet actionnaire suite à l'engagement de ses fonds dans le contrôle de son dirigeant, ce qui dote ce dernier de larges marges de manœuvres pour esquisser la stratégie de l'entreprise ainsi que l'affectation de ses cash-flows (Charreaux 1997) $^{6}$. Ce type de contrôle par le cours de l'action, favorisé par un actionnariat fortement éclaté, peut difficilement constituer un barrage à une éventuelle OPA ce qui pousse les dirigeants, à centrer leur stratégie vers la recherche de la maximisation de la rentabilité financière de la firme.

\section{Les modèles des effets du dividende sur la valeur de l'entreprise}

Modigliani et Miller (1961) ont constitué par leurs travaux dans l'hypothèse d'un marché parfait, un cadre référentiel de politique de dividende qui n'a aucun impact sur la valeur de la firme, connu sous le titre de thèse de neutralité de la politique de dividende, l'absence d'une influence de cette politique sur le cours boursier, est soutenue par (Black et Scholes 1974; Poulain-Rehm 2005).

\section{L'effet neutre ou thèse de neutralité :}

En (1961), Modigliani et Miller confirmaient l'absence de toute possibilité de valoriser ou de dévaloriser une firme, respectivement à partir de l'importance ou de la faiblesse de ses dividendes distribués, tant que toutes les transactions se fassent dans un marché financier parfait, les hypothèses sous-jacentes à cette neutralité de la politique de dividendes peuvent être résumées ainsi :

$\checkmark$ Il n'y a pas de coût de transaction pour les investisseurs, qui désirent convertir l'amélioration du cours du titre en plus-value.

$\checkmark \quad$ L'entreprise qui distribue trop de dividendes peut émettre des actions sans coût de transaction, et sans frais d'émission, en investissant dans des projets rentables

$\checkmark$ Dans les entreprises qui ne versent pas assez de dividendes, les dirigeants ne peuvent ni utiliser les excédents dans leur propre intérêt, ni les affecter à de mauvais projets d'investissement

$\checkmark$ Le taux d'imposition des dividendes et des plus-values est le même.

En publiant en octobre 1961 dans «the journal off business », leur article qui neutralise la politique de distribution, Franco Modigliani \& Milton Miller, donnaient le feu vert aux chercheurs de la finance à construire une

${ }^{6}$ Charreaux G., « Modes de contrôle des dirigeants et performance des firmes », (Charreaux, 1997). Cité dans Morin, F., \& Rigamonti, E. (2002). 
multitude de théories visant à expliquer l'impact que peut avoir un tel choix de politique de dividendes sur la valeur de la firme, notamment avec la progression du rôle du marché financier en tant que régulateur économique et déterminant des valeurs des firmes cotées. Pour ces deux auteurs, dans un marché financier parfait (caractérisé par la transparence, l'absence des coûts de transactions, disponibilité de l'information...), la politique de dividendes adoptée par une firme est neutre vis-à-vis de sa valeur sur le marché, autrement dit, que l'entreprise distribue de dividendes ou renforce ses capitaux propres est une opération indifférente pour l'actionnaire, leur raisonnement s'appuie sur le fait qu'un investisseur rationnel, dans une hypothèse d'égalité de l'impôt retenu dans les deux cas, que ce soit la réception des dividendes ou la réalisation de plus-value en capital, suite à une cession de ses titres, réalisera le même gain, car toute modification du montant de dividende à recevoir se trouve compensée par un ajustement de la valeur de l'action, ce raisonnement rejoint l'idée de Walter (1956), qui considérait que la décision de distribution est une décision résiduelle, n'ayant lieu qu'après celle d'investissement et de financement. Cependant Graham et Dodd (1951), affirmaient que la réception d'un dollar en dividendes impacte quatre fois de plus le cours de l'action qu'un dollar mis en réserves.

\subsection{Le modèle de neutralité de Modigliani et Miller (1961)}

Dans un marché financier parfait ${ }^{7}$ où il n'y a pas de différence d'imposition des dividendes et des plus-values sur la cession des actions, de sorte que la fiscalité n'impacte pas les arbitrages tant qu'il n'y a qu'un seul taux d'impôt applicable sur le revenu des titres, que ce revenu soit un dividende ou une plus-value sur cession de ce même titre (Stiriba 2014). Modigliani et Miller (1961) en mettant d'autres hypothèses très théoriques, ont démontré que la politique de dividende n'a pas d'impact sur la valeur boursière des actions de la société.

Le modèle de $M \& M$ peut se démontrer par formules comme suit :

$$
\text { Soit } \quad \mathbf{V}=\mathbf{P}_{\mathbf{t}} \mathbf{N}_{\mathbf{t}}
$$

$\mathrm{V}$ : valeur de marché des actions de la société ou valeur boursière de la société. $\mathrm{Pt}$ : prix de l'action à la date $\mathrm{t}$

$\mathrm{Nt}$ : nombre des actions à la date $\mathrm{t}$

Si le prix P d'une action égale la somme des flux qu'elle pourra générer dans le futur et qui sont le dividende à recevoir et le prix de cession de cette action, actualisés au taux de rentabilité (r) exigé par les actionnaires, donc on peut écrire :

$$
\mathbf{P}=\left(\mathbf{P}_{t+1}+\mathbf{D I V}_{t}\right) /(\mathbf{1}+\mathbf{r})
$$

${ }^{7}$ Les caractéristiques du marché parfait : des taux de prêt et d'emprunt sont identiques, une absence de coûts de transaction, la transparence dans l'information - et une négligence de l'impact de la fiscalité. 
$r$ : taux de la rentabilité sur l'investissement en action.

L'équilibre financier de l'entreprise entre les emplois et les ressources de la date $(\mathrm{t})$ à la date $(\mathrm{t}+1)$ est déterminé par l'équation suivante :

$I_{N V_{t}}+\mathbf{N}_{\mathbf{t}} \cdot \mathbf{D I V}_{\mathbf{t}}+\mathbf{F R N}_{\mathbf{t}}=\mathbf{C A F}_{\mathbf{t}}+\mathbf{n} \cdot \mathbf{P}_{\mathbf{t}+\mathbf{1}}+\mathbf{E}_{\mathbf{t}}$

$\mathrm{INV}_{\mathbf{t}}$ : Montant des investissements nets de la période ;

$\mathrm{N}_{\mathbf{t}}$.DIV $\mathbf{t}$ : Montant des dividendes versés ;

$\mathrm{FRN}_{\mathrm{t}}$ : Variation du fonds de roulement net ;

$\mathrm{CAF}_{t}$ : Capacité d'autofinancement de la période ;

n. $\mathrm{Pt}_{+1}$ : Montant de l'augmentation de capital (émission de $\mathrm{n}$ actions nouvelles au prix $\left.P_{t+1}\right) E_{t}$ : Endettement net sur la période.

A partir de cette équation on peut faire ressortir : $\mathrm{n}$. $\mathrm{P}_{\mathbf{t}+\mathbf{1}}$

n. $P_{t+1}=I N V_{t}+N_{t} \cdot D I V_{t}+F_{R} N_{t}-C A F_{t}-E_{t}$

Le nombre d'actions à la date $(\mathrm{t}+1)$, noté $\mathrm{N}_{\mathbf{t}+\mathbf{1}}$ sera $\mathrm{N}_{\mathbf{t}+\mathbf{1}}=\mathrm{n}+\mathrm{N}_{\mathrm{t}}$ d'où $\mathbf{N}_{\mathbf{t}}=\mathbf{N}_{\mathbf{t}+\mathbf{1}}$

$-\mathbf{n}$

On remplace (5) dans (1) pour déterminer (V) la valeur des actions de la société sur le marché boursier, alors on écrit :

$\mathbf{V}=\mathbf{P}_{\mathbf{t}} \cdot \mathbf{N}_{\mathbf{t}}=\mathbf{P}_{\mathbf{t}}\left(\mathbf{N}_{\mathbf{t}+\mathbf{1}}-\mathbf{n}\right)$ : on obtient (6) et on remplace (2) dans (6) la valeur devient :

$\mathbf{V}=\mathbf{P}_{\mathrm{t}} . \mathbf{N}_{\mathrm{t}}=\operatorname{Pt}\left(\mathbf{N}_{\mathrm{t}+1}-\mathbf{n}\right)=\left(\mathbf{P t}_{+1}+\mathbf{D I V}_{\mathbf{t}}\right)\left(\mathbf{N}_{\mathbf{t}+1}-\mathbf{n}\right) /(\mathbf{1}+\mathbf{r})$

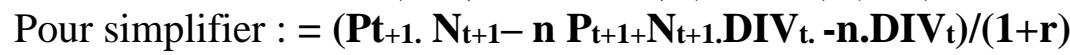

$$
\begin{aligned}
& =\left(\mathbf{P t}_{+1} . \mathbf{N}_{\mathrm{t}+1}-\mathbf{n} \mathbf{P t}_{+1+}\left(\mathbf{N}_{\mathrm{t}+1}-\mathrm{n}\right) \mathrm{DIV}_{\mathrm{t}}\right) /(\mathbf{1}+\mathbf{r}) \\
& =\left(\mathbf{P t}_{+1} . \mathbf{N}_{\mathrm{t}+1}-\mathbf{n} \mathbf{P t}_{+1+} \mathbf{N}_{\mathrm{t}} . \mathbf{D I V} \mathrm{V}_{\mathrm{t}}\right) /(\mathbf{1}+\mathbf{r})
\end{aligned}
$$

On remplace (4) dans (7), alors on trouve :

$$
\begin{aligned}
& =\left(\mathbf{P t}_{t+1} . \mathbf{N}_{\mathrm{t}+1-1}-\mathrm{IV}_{\mathrm{t}}-\mathrm{FRN}_{\mathrm{t}}+\mathrm{CAF}_{\mathrm{t}}+\mathrm{E}_{\mathrm{t}}\right) /(\mathbf{1}+\mathrm{r}) \\
& =\left(\mathbf{P t}_{+1} . \mathbf{N}_{\mathrm{t}+1}+\mathbf{F R N}_{\mathrm{t}}-\mathrm{INV}_{\mathrm{t}}-\mathrm{CAF}_{\mathrm{t}}-\mathbf{E}_{\mathrm{t}}\right) /(\mathbf{1}+\mathbf{r})
\end{aligned}
$$

D'après cette équation, Modigliani et Miller (1961), concluent que la valeur de l'entreprise ne dépend plus de la politique de distribution de dividendes, comme on peut le remarquer l'équation ne comporte plus les variables de la politique de dividendes car ces dernières se sont simplifiées. Plutôt elle dépend selon les auteurs du rendement et de l'efficacité des investissements, toutefois les hypothèses mises auparavant sont loin de la réalité, prenons par exemple celle relative à l'imposition et qui considère que les dividendes et les plus-values de cession sont imposés au même taux, est une condition fictive, cependant les auteurs étaient conscients de cette réalité d'inefficience des marchés mais ils voulaient montrer comment un choix de financement purement théorique peut se faire sur un marché, et pour qu'un modèle soit logiquement structuré, ils préféraient le marché parfait. Leur modèle constituait un point de départ d'apparition d'une large littérature enrichie de théories cherchant à expliquer les décisions financières. 


\subsection{Le modèle de Black et Scholes (1974)}

Le modèle Black et Scholes (1974) s'inscrit dans la validation et la confirmation des travaux de Modigliani et Miller (1961), ce modèle se présente de la manière suivante :

$$
R_{i t}=c_{1}+\left(R m-c_{1}\right) \beta_{i t}+c_{2}\left(d_{i t}-d_{m t}\right) / d_{m t}+\varepsilon_{i t}
$$

$\mathbf{R}_{\text {it }}$ : Taux de rentabilité (ou rendement) espéré du portefeuille i à la date $t$ $\mathbf{c}_{1}$ : Ordonnée à l'origine qui, d'après le MEDAF devrait correspondre au taux d'intérêt sans risque

$\mathbf{R}_{\mathbf{m}}$ : taux de rentabilité (ou rendement) espéré du portefeuille de marché à la date $\mathrm{t}$

$\beta_{i t}$ : Coefficient de risque systématique du portefeuille i à la date $\mathrm{t}$ c2 : coefficient mesurant l'impact des dividendes sur la rentabilité du portefeuille

dit : Taux de rendement du dividende du portefeuille $\mathrm{i}$, qui correspond à la somme des dividendes reçus au cours de 12 mois qui précèdent la date $t$, qui est ensuite rapportée à la valeur du portefeuille à la fin de l'année

$\mathbf{d}_{\mathbf{m t}}$ : Taux de rendement du dividende du portefeuille de marché, mesuré sur les 12 mois qui précèdent la date $t$

$\varepsilon_{i t}$ : Terme d'erreur.

Sur un échantillon composé de 25 portefeuilles, ciblant 5 catégories de taux de rendement, l'étude de Black et Scholes (1974) avait comme objectif la vérification de l'existence d'une telle relation entre le rendement de l'action (dividende) et la rentabilité du portefeuille ( $\left.\mathbf{R}_{\mathbf{i t}}\right)$, pour ce faire, ils ont retenu les cours mensuels et les dividendes de toutes les actions cotées au New York Stock Exchange de janvier 1936 à mars 1966. Ils avaient pour clés de trancher dans l'impact de la politique de dividendes sur la valeur de la firme à partir du coefficient $\left(c_{2}\right)$, ainsi pour pouvoir dire qu'il y a une influence, il faut que l'indicateur $\left(\mathrm{c}_{2}\right)$ soit différent de 0 . Cependant les auteurs affirment qu'il n'a jamais été différent de zéro, la rentabilité des portefeuilles $\left(\mathbf{R}_{\mathbf{i t}}\right)$ était presque la même que ce soit pour les actions à taux de rendement élevé des dividendes ou pour ceux à taux de rendement faible, ce qui laisse déduire que la distribution de dividendes n'affecte pas la valeur de la firme.

\section{La remise en cause de la neutralité du dividende}

2.1. Remise en cause par la présence d'aversion au risque et d'incertitudes des flux

Remettre en cause ou infirmer la neutralité du dividende, c'est montrer théoriquement et/ou empiriquement que le niveau de la distribution de dividendes exerce certains effets sur la valeur des actions. Ces effets peuvent conduire la firme à mener certains comportements financiers. La compréhension de ces comportements renvoie généralement, dans ce domaine 
à parcourir les écrits de Walter (1956), Lintner (1956), Gordon (1959), (Graham-Dodd (1951), Stiriba (2013). Dans ce paragraphe on discutera les apports de ces chercheurs dans l'explication de l'impact de la distribution de dividende sur la valeur des actions. Signalons ici que le modèle de Lintner a été attaché en haut à la quasi-stabilité du dividende, confirmant à son tour l'effet indiscutable du niveau du dividende sur l'évaluation que peut émettre un actionnaire sur les actions d'une firme à travers la progression du dividende.

\subsubsection{Le modèle de Gordon 1956}

Graham et Dodd (1951) proposaient qu'un dollar de dividende reçu en main suite à la possession d'une action, a en moyenne un effet sur les cours boursiers de cette action quatre fois supérieur à celui qu'un dollar mis en réserve par la firme. C'est la théorie connue sous «l'oiseaux en main », “ the Bird in the Hand" (BIH), l'actionnaire préfère un dividende certain à une plusvalue hypothétique. Dans cette même analogie de raisonnement, les travaux de Gordon et Shapiro en (1956), ont permis le développement d'une nouvelle théorie pour évaluer les actions. Cette fois non plus à partir des données historiques mais à partir de l'actualisation des rendements futurs que peut produire un titre, sans prendre en compte les plus-values de cession. En effet, si le taux de rendement exigé par les actionnaires augmente avec le risque des flux monétaires et que l'incertitude augmente avec l'échéance éloignée, toutes choses étant égales par ailleurs, les actionnaires ayant un risque et une incertitude élevée à l'égard des flux monétaires du titre vont exiger un taux de rendement important. Chose qui poussera la firme à verser plus de dividendes afin d'éviter la hausse du taux d'actualisation (taux de rendement exigé par les actionnaires). Cela conduit à la relation suivante : il faut distribuer un dividende élevé pour un taux d'actualisation élevé, ce qui augmente le prix de l'action, d'où l'influence de dividende sur la valeur de l'action.

Le modèle de Gordon et Shapiro peut se présenter ainsi :

Soit $\left(\mathrm{P}_{0}\right)$ le prix d'une action à la date $\mathrm{t}_{0}$

$\left(D_{t}\right)$ Dividende versé à la date $t$

(K) Le taux d'actualisation qui correspond au taux de rendement exigé.

Alors la valeur de l'action $\left(\mathrm{P}_{0}\right)$ à la date $\mathrm{t}_{0}$ est :

$$
\left(\mathrm{P}_{0}\right)=\sum_{t=1}^{n}\left(\frac{(\mathrm{Dt})}{(1+k)^{t}}\right)
$$

Cette formule implique que la valeur d'un titre est égale à la somme d'une suite de dividendes dont les prévisions sont difficiles, pour remédier à ce problème mathématique, Gordon et Shapiro (1956) ont pensé à l'hypothèse qui admet que le dividende croit à un taux (g) qui est inférieur au taux d'actualisation (k) exigé par l'actionnaire, ce qui simplifie la formule précédente et la rend de la forme : 


$$
\mathrm{PO}=\frac{\mathrm{D} \mathbf{1}}{\mathrm{K}-\mathrm{g}}
$$

Cependant cette conception a suscité plusieurs critiques au niveau empirique, ainsi Shiller ${ }^{8} 1981$ avance qu'il est difficile d'estimer le taux d'actualisation ( $\mathrm{k}$ ) et d'anticiper le taux de croissance des dividendes futurs à long terme (g), Higgins (1972) encore critique cette conception dans la mesure où Gordon suppose une séparation entre la politique de dividendes et celles d'investissement et de financement, pour Higgins $(1972)^{9}$ la distribution des dividendes est un produit dérivé de ceux d'investissement et de financement.

\subsubsection{La théorie résiduelle des dividendes : le modèle de Walter (1956)}

La conception de Walter 1956 arrive parmi les principales critiques adressées à l'encontre de la théorie de Modigliani et Miller (1961), surtout en ce qui concerne les hypothèses d'un marché parfait, Walter 1956 démontrait que la politique de dividende influence de façon directe la valeur de la firme, Walter revoit la politique de dividende comme le résultat d'une décision de financement, déterminant le taux de rétention des bénéfices sur la base des besoins à financer par les ressources internes, dont le bénéfice de l'exercice fait partie déterminante, pour l'auteur, le dividende n'est rien d'autre qu'un paiement d'un solde résiduel (Taleb 2019), d'où la politique de dividende est une décision résiduelle, qui vient comme décision dérivée de la décision de financement global de la firme, encore pour l'auteur, la distribution de dividende est fonction des opportunités d'investissement offertes à l'entreprise. En présence des investissements rentables, les flux de trésorerie seront drainés vers le financement de ces projets, lorsque ces derniers ne seront pas jugés financièrement rentables, les flux de trésorerie feront l'objet d'une distribution de dividendes, de sortes que les ratios de rétention ou de distribution varient entre $0 \%$ et $100 \%$, comparaison faite entre le taux de rentabilité interne des investissements et le taux pour disposer du capital (le coût du capital), il n'y aura distribution de dividendes qu'au cas où le coût du capital soit supérieur au taux de rentabilité des investissements, le cas contraire c'est l'investissement qui prime.

Le modèle de Walter, sous certaines hypothèses ${ }^{10}$ peut se présenter comme suit : Soit $\mathrm{V}_{\mathbf{c}}$ la valeur de marché.

${ }^{8}$ Consulté dans Taleb, L. (2019). Politique de distribution de dividendes dans le cadre d'un marché parfait : Une revue de la littérature. P.4

9 Consulté dans Taleb, L. (2019). Politique de distribution de dividendes dans le cadre d'un marché parfait : Une revue de la littérature. P.4

${ }^{10}$ Walter suppose pour la dérivation de sa formule que :

1) Le taux de capitalisation k exigé par le marché est constant

2) Le financement de l'investissement s'effectue en priorité par autofinancement

3) Le dividende par action ne doit en aucun cas dépasser le bénéfice par action. 
D est le dividende par action

$B$ est le bénéfice par action

$\mathrm{R}_{\mathbf{a}}$ est le taux de rentabilité des investissements

$\mathrm{R}_{\mathbf{c}}$ est le taux de rentabilité exigé par le marché.

$$
v_{C}=\frac{D+\frac{R_{a}}{R c}(B-D)}{R c}=\frac{B}{R c}+\frac{R a-R c}{(R c * R c)}(B-D)
$$

Cette formule permet selon Walter d'établir une politique de dividende optimale, qui peut maximiser la valeur de l'action et donc la valeur de la firme. Cette conception a été largement critiquée, dans le point où la société peut simultanément distribuer des dividendes et procéder à l'augmentation du capital par émission d'actions nouvelles, ce qui permet au dividende de dépasser le bénéfice par action, chose qui contredit la troisième hypothèse du modèle de Walter, d'une part, d'autre part, considérer la politique de dividendes comme une décision résiduelle déterminée uniquement par l'absence ou la présence des opportunités d'investissement, est contradictoire eu égard à la réalité des entreprises qui, comme le confirment Lintner (1956) et plus récemment Brav et al. (2003), cherchent de manière continuelle à adopter une distribution quasi stable, voir légèrement concrétisant une tendance à la hausse.

\subsection{Remise en cause de la neutralité par la présence des Conflits d'intérêt}

A l'inverse de la théorie financière néoclassique, la théorie d'agence, analyse l'entreprise à l'image d'un nœud de contrats entre les agents (salariés dirigeants) et les principaux (les propriétaires), qui vont déléguer la prise de décisions et la gestion à ces agents. Suite à ce pouvoir discrétionnaire dont vont disposer les dirigeants, chacun va chercher à réaliser ses propres intérêts privés, même au détriment de l'autre. Ce qui conduit à des conflits d'intérêts. Les actionnaires, de leur part, pour défendre leurs intérêts, doivent mettre en place des mécanismes de contrôle, ils doivent donc supporter des couts appelés « coûts d'agence », dans ce cadre, Jensen et Meckling (1976) en se référant aux travaux de Fama et Miller (1972), définissent deux catégories de conflits d'intérêt engendrés par la distribution de dividende, il s'agit du conflit actionnaire/dirigeant et du conflit actionnaire/créancier.

\subsubsection{Conflit d'intérêt entre actionnaires et dirigeants}

Dès l'arrivée des travaux de Berle et Means (1932) ${ }^{11}$, l'économie enregistrait un nouveau mode de gestion de ses affaires, caractérisé pour la

${ }^{11}$ Charreaux, G. (2000). Le conseil d'administration dans les théories de la gouvernance. Revue du financier, 127, 6-17. 
première fois par une séparation entre la propriété et le contrôle. Dès lors, les relations entre agent et mandataire vont déboucher sur une large suite de recherches inspirées au départ des travaux de Jensen et Meckling (1976) fondateurs de la théorie d'agence, cette séparation donnerait lieu à une concrétisation de la divergence d'intérêts entre le dirigeant et le propriétaire. Divergence qui était implicite, mais une fois le dirigeant s'est approprié de quelques pouvoirs discrétionnaires, la préférence de ses propres intérêts à ceux des actionnaires propriétaires de la firme dont il est agent, s'est mise en place via plusieurs comportements. En effet l'actionnaire cherchant à préserver ses intérêts, va chercher à mettre en place plusieurs mécanismes de gouvernance, visant à surmonter les problèmes et défis résultant d'asymétrie d'information, justifiés par l'antisélection et l'aléa moral que peuvent entreprendre les comportements de l'agent. Le dividende figure parmi les principaux mécanismes mis à la disposition du principal afin de discipliner son agent. Ainsi exiger de l'agent un niveau de dividende donné est une technique de le contraindre à être efficace et efficient, et à rentabiliser les capitaux mis à sa gestion, via un benchmarking fait avec le rendement des actions équivalentes sur le marché par type d'activité ou par secteur ou en général sur le marché financier. Ce mécanisme de gouvernance (le dividende) fixé par le principal afin de discipliner son agent, exige que les flux nets de trésorerie soient toujours à distribués aux actionnaires, et que le financement des futurs investissements se fasse par les dettes, et comme ces dettes doivent être rémunérées sous forme des intérêts, l'agent se trouve dans l'obligation de générer des rendements des actions. Ainsi donc, les fonds que l'agent peut mobiliser dans des activités maximisant ses intérêts privés se trouvent absorbés par la distribution du dividende, tout cela se passe dans une entreprise managériale. Lorsque la firme devient non managériale le conflit entre principal et dirigeant se transforme à un conflit entre le principal et le manager, ce dernier cherchera à adopter des stratégies selon sa part dans le capital, son comportement devient aligné sur les intérêts d'autres actionnaires mais après une détention significative dans le capital. Ces comportements ont fait l'objet des travaux de plusieurs auteurs, cependant comme l'évoque Charreaux (1997) trop de contrôle peut nuire à la gestion et réduire les capacités discrétionnaires du dirigeant. Le conflit entre les actionnaires eux-mêmes sur le dividende, trouve son explication dans les horizons de chacun d'entre eux, si les minoritaires préfèrent souvent la distribution alors les majoritaires au contraire préfèrent la rétention et le réinvestissement puisqu'ils ont une vision des rendements de long terme. Le conflit sur le dividende, nait donc du fait qu'il peut constituer un objectif ultime pour certaine catégorie d'actionnaires comme les minoritaires et les actionnaires de bloc qui souhaitent une distribution immédiate, inversement à certaines autres catégories d'actionnaires tels que les actionnaires familiaux ou majoritaires qui préfèrent 
le réinvestissement des cash-flows. Le dirigeant peut profiter de cette divergence d'intérêts en cherchant à constituer des réseaux avec certains actionnaires ou certaines firmes avec lesquelles l'entreprise effectue des transactions financières ou économiques. Ces réseaux sont spécifiques à des buts privés de cette coalition de manière analogue, on parle ici des stratégies d'enracinement de l'agent. A l'image de cette divergence d'intérêts entre les actionnaires, le dirigent à son tour va préférer le réinvestissement des cashflows dans des projets difficilement imitables et dont la maitrise de leurs techniques demande plus de temps. En général, il va investir de manière à ce que son remplacement par un autre dirigeant devient difficile voire impossible.

\subsubsection{Conflit d'intérêt entre actionnaires et créanciers}

Les cash-flows disponibles en trésorerie de l'entreprise constituent un moyen de financement, si sa distribution sous forme de dividende, sera compensée par l'endettement cela peut pousser les créanciers à avoir peur sur leurs fonds, et ce par crainte et aversion au risque de faillite et de ne pas pouvoir recouvrir leurs créances. Cette crainte de perte éventuelle de la part des prêteurs peut être révisée à la baisse en exigeant des clauses de garanties dans les contrats de prêt. Le montant du dividende, vient donc à côté d'autres facteurs renforcer l'aversion au risque et qui alimentent les conflits entre ces deux parties. Si les actifs constituent la garantie des créances, la distribution excessive de cash remplacée alors par les emprunts conduira à une diminution de la valeur des obligations de telle sorte que la valeur de l'obligation se trouve négativement associée au niveau de distribution de dividende car, une grande part de l'actif est financée par les dettes, et pour que cette idée soit de plus en plus assimilable, imaginons le cas extrême où la totalité de l'actif est financée par les dettes et que l'entreprise décide de céder son actif ou subira par quelconque motif, une liquidation, alors il devient simple de comprendre que les créances se trouvent alors sans garantie, d'où une forte réticence des créanciers à l'égard de toute politique de dividende dite généreuse et par conséquent la naissance du conflit actionnaires/créanciers.

Dans la norme c'est le bénéfice distribuable qui constitue l'origine du dividende, et le niveau de distribution doit être fonction du niveau des rendements réalisés par l'entreprise, sans réduire les fonds drainés vers les investissements. La distribution de dividendes ne doit en aucun cas être financée par des émissions d'emprunts ou cession d'actifs, car la valeur de l'action est fonction de la valeur créée par les actifs, et toute destruction des actifs conduit également à la chute des valeurs des titres de créances, et afin de limiter les effets négatifs de ces modalités de financement de la distribution de dividendes, les créanciers sont invités à renforcer les clauses protectrices de leurs droits dans les contrats de prêt consentis à la firme, ces clauses sont censées désigner et déterminer, les types ou modes de financements auxquels 
la firme peut recourir, pour financer la distribution des dividendes à ses actionnaires.

\subsection{Remise en cause par le rôle informationnel du dividende}

La conception de Modigliani et Miler (1961), qui exclue toute influence de la politique de dividendes sur la valeur de la firme, a été critiquée par plusieurs travaux traitant en même temps, le rôle informatif que peut jouer le dividende, la signalisation par le dividende, l'effet fiscal et l'effet clientèle. La théorie d'efficience informationnelle constitue le noyau dur de la théorie financière moderne, l'hypothèse fondamentale de cette théorie est que les titres possèdent une valeur dite «valeur fondamentale », qui n'est rien d'autre que la somme des flux futurs que génèrera un titre. Lorsqu'il s'agit d'une action, ces revenus futurs sont donc les dividendes auxquels donnera lieu l'action, et comme ces dividendes futurs sont inconnus, les actionnaires doivent les anticiper à partir des informations dont ils disposent, d'où l'impact du dividende sur la valeur de la firme (Lardic \& Mignon 2006).

\section{Conclusion}

Cet article a essayé, via la littérature de la finance d'entreprise, de faire le point des travaux antérieurs, dans la mesure du possible, sur certaines notions relatives à la distribution de cash-flows, notamment le dividende, le rachat d'actions, ainsi que les interactions qui peuvent surgir entre un tel mode de distribution et la structure d'actionnariat (nature d'actionnariat et structure du capital). En conclusion, le dividende constitue une variable qui est prise en compte dans tous les actes (financement, partage de la valeur ajouté...) des intervenants de la vie de la firme (actionnaire, manager, créancier...). La nature ou la qualité d'actionnaire influence la distribution de manière non exhaustive, et en fonction du pourcentage de capital détenu et ouvrant droit au contrôle de la firme, et in fine la problématique de la politique de dividende n'a pas encore reçu une réponse unanime au travers les études que ce soient théoriques ou empiriques, puisqu'il n'y a pas de modèle universel déterminant le montant du dividende à distribuer par une firme. Cela peut s'expliquer par l'existence d'une variable non négligeable en science de gestion, à savoir « le contexte », cette variable incarne à son intérieur plusieurs facteurs (économiques, sociaux, environnementaux, politiques, culturels, degré de la gouvernance...), qui constituent donc des sujets de recherche, et qui ne sont pas les mêmes au travers les pays lieux des études. Ce qui débouche sur une divergence entre les résultats tirés des études réalisées.

\section{References:}

1. Abou El Jaouad., M. (2010), Fiscalité appliquée de l'entreprise Marocaine, les Editions Maghrébines. 
2. Adjaoud, F. Zéghal, D. (1993). Fiscalité et politique de dividende au Canada : nouveaux résultats. Fineco, 3(1), 143-151.

3. Adjaoud, F., Chkir, I et Rahman. A. (2005). Dividende courant et bénéfice à venir universitéd'ottawa. finéco, vol. 15 . P : 81

4. Albouy, M. (1990). La politique de dividendes des entreprises. Revue d'économie financière, (12/13), 240-251.

5. Albouy, M., et Morris, T. (2006). Les rachats d'actions au Canada : motivations et impact de l'activité économique. Finance Contrôle Stratégie, 9(4), 5-32.

6. Atindéhou, R. (1996). La variation inattendue du dividende régulier : un signal d'évolution du bêta ? Fineco. P 71-88.

7. Benzaouagh, M., et Tebbaa, J. E. (2016). Signalisation Par Le Rachat d'actions : Cas Des Societes Cotées A La Bourse De Casablanca. Global Journal of Management And Business Research.

8. Black, F. (1976), The dividend puzzle, Journal of Portfolio Management, 2(1): 5-8.

9. Black, F., et Scholes, M. (1974). The effects of dividend yield and dividend policy on common stock prices and returns. Journal of financial economics, 1(1), 1-22.

10. Brav, A., Graham, J., Harvey, C., Michaely, R. (2005). "Payout policy in the 21st century". Journal of Financial Economics, 77(3), pp 483527.

11. Charreaux, G. (1991). Structures de propriété, relation d'agence et performance financière Ownership structures, agency relationship and financial performance. Revue économique, 521-552.

12. Charreaux, G. (2000). Le conseil d'administration dans les théories de la gouvernance. Revue du financier, 127, 6-17.

13. Damodaran A, (2010), Finance d'entreprise. Théorie et pratique, DeBoek.

14. Demsetz, H., Lehn, K., (1985). The structure of corporate ownership: Causes and consequences, Journal of Political Economy 93(6): 11551177.

15. Desbrières, P., Saint-Onge, S., et Magnan, M. (2000). Les plans d'option sur actions : théorie et pratique. Les rémunérations-Politiques et pratiques pour les années 2000, 135-160.

16. Easterbrook, F. (1984). Two agency-cost explanations of dividends. The American Economic Review, 74, 650-659.

17. El Houcine, R., et Boubaker, A. (2015). La relation entre le rachat d'actions et la politique des stock-options salariales. Recherches en Sciences de Gestion, (2), 67-83. 
18. Faccio M. et Lang L. (2002), «The Ultimate Ownership of Western European Corporations », Journal of Financial Economics, vol. 65, ${ }^{\circ}$ 3, p. 365-395.

19. Fama E.F. et Jensen M.C., 1983, « Separation of Ownership and Control », Journal of Law and Economics, vol. 26, June, p. 301-326.

20. Frankfurter, G., Wood, B. G., et Wansley, J. (2003). Dividend policy: Theory and practice. Elsevier.

21. Gadhoum, Y. (1995). L'actionnariat canadien : analyse statistique et comparaison internationale. Finance, Economie et Comptabilité, 5, 1.

22. Gordon, M. J., et Shapiro, E. (1956). Capital equipment analysis: the required rate of profit. Management science, 3(1), 102-110.

23. Graham, B., et Dodd, D. (1951). Security Analysis. New York : McGraw-Hill.

24. Grullon, G., et Ikenberry, D. L. (2000), "What do we know about stock repurchase", Journal of Applied Corporate Finance, 13(1), pp. 31-51.

25. Jensen, M. C., et Meckling, W. H. (1976). Theory of the firm: Managerial behavior, agency costs and ownership structure. Journal of financial economics, 3(4), 305-360.

26. Jonathan B., Peter D. (2011). Finance d'entreprise. Pearson France.

27. Kahle, K. M. (2002). When a buyback isn't a buyback: Open market repurchases and employee options. Journal of Financial Economics, 63(2), 235-261.

28. Lamyaa, Riad., et Touili, K. (2017). Pourquoi choisir le rachat d'actions ? Revue du contrôle, de la comptabilité et de l'audit, 1(3).

29. Lardic, S., et Mignon, V. (2006). L'efficience informationnelle des marchés financiers. La découverte.

30. Lawriwsky, M.L. (1984): Corporate Structure and Performance, St. Martin's Press, New York.

31. Lintner J., (1956) .Distribution of Incomes of Corporations Among Dividends, Retained earnings, and taxes. American Economic Review 61, N 2. 97-113.

32. Mard, Y., Marsat, S., et Roux, F. (2014). Structure de l'actionnariat et performance financière de l'entreprise : le cas français. Finance Contrôle Stratégie, (17-4).

33. Morin, F., et Rigamonti, E. (2002). Évolution et structure de l'actionnariat en France. Revue française de gestion, (5), 155-181.

34. Mueller, D. C. (1972). A life cycle theory of the firm. The Journal of Industrial Economics, 199-219.

35. Nazih. A (2014). Entreprises familiales Mieux parées contre les crises. www.leconomiste.com

36. Poulain-Rehm, T. (2005). L'impact de l'affectation du free cash-flows sur la création de valeur actionnariale : le cas de la politique 
d'endettement et de dividendes des entreprises françaises cotées. Finance Contrôle Stratégie, 8(4), 205-238.

37. Shamsidine, A. A. (2001). L'achat par la société de ses propres actions. Revue internationale de droit économique, 15(1), 41-76.

38. Shleifer A. et Vishny R.W. (1986), "Large Shareholders and Corporate Control », Journal of Political Economy, vol. 94, 3, p. 461489.

39. Shleifer, A., et Vishny, R. W. (1997). A survey of corporate governance. The journal of finance, 52(2), 737-783.

40. Stiriba. latifa, (2013). Thèse : Décision de financement, politique de dividendes et création de la valeur : Essai de modélisation

41. Taleb, L. (2015). Politique de Dividende des Entreprises Tunisiennes : Une Explication par une Etude de Cas et les Modèles de Base (Dividend Policy of Tunisian Firms : An Explanation with a Case Study and Basic Models).

42. Taleb, L. (2019). Politique de distribution de dividendes dans le cadre d'un marché parfait : Une revue de la littérature (Dividend Policy in Perfect Market: A Literature Review). Available at SSRN 3313799.

43. Trigo Trindade, R., \& Bahar, R. (2002). Droits des actionnaires minoritaires en Suisse. In Rapports suisses présentés au XVIème Congrès international de droit comparé Swiss reports presented at the XVIth International Congress of Comparative Law (381-458).

44. Turki, A. (2013). Le rôle de la politique de dividendes dans le cadre des opérations de fusions-acquisitions (Doctoral dissertation). HAL.Archives Ouvertes.

45. Vermaelen, T. (2006). Share Repurchases Can Be a Good Deal,". Financial Times, 19, 2.

46. Vernimmen., P. (2014). Finance de l'entreprise, Dalloz. ;839-840.

47. Walter (J.E.), 1956 « Dividend Policy and Common Stock Prices », Journal of Finance, vol.11, pp. 29-41

48. Wang, D. (2006). Founding family ownership and earnings quality. Journal of Accounting Research 44 (3) : 619-655.

49. Weisbenner, S. J. (2000). Corporate share repurchases in the 1990s: What role do stock options play? dans AFA 2002 Atlanta Meetings.

50. Yessoufou, A, R. (2018). Signalisation du risque à l'annonce du dividende dans le contexte africain : une étude empirique sur la BRVM, Revue D'Etudes en Management et Finance D'Organisation $n^{\circ} 7$. 